\title{
Thopaz+ Portable Digital System for Managing Chest Drains: A NICE Medical Technology Guidance
}

\author{
James Michael Evans ${ }^{1} \cdot$ Alistair Ray ${ }^{1} \cdot$ Megan Dale $^{2} \oplus \cdot$ Helen Morgan $^{1} \cdot$ Paul Dimmock $^{3} \cdot$ Grace Carolan-Rees $^{2}$
}

Published online: 23 January 2019

(c) The Author(s) 2019

\begin{abstract}
The Thopaz+ portable digital system was evaluated by the Medical Technologies Advisory Committee (MTAC) of the National Institute for Health and Care Excellence (NICE). The manufacturer, Medela, submitted a case for the adoption of Thopaz+ that was critiqued by Cedar, on behalf of NICE. Due to a lack of clinical evidence submitted by the manufacturer, Cedar carried out its own literature search. Clinical evidence showed that the use of Thopaz+ led to shorter drainage times, a shorter hospital stay, lower rates of chest drain re-insertion and higher patient satisfaction compared to conventional chest drainage when used in patients following pulmonary resection. One comparative study of the use of Thopaz+ in patients with spontaneous pneumothorax was identified and showed shorter drainage times and a shorter length of hospital stay compared to conventional drainage. No economic evidence was submitted by the manufacturer, but a simple decision tree model was included. The model was improved by Cedar and showed a cost saving of $£ 111.33$ per patient when Thopaz+ was used instead of conventional chest drainage in patients following pulmonary resection. Cedar also carried out a sub-group analysis of the use of Thopaz+ instead of conventional drainage in patients with pneumothorax where a cost saving of $£ 550.90$ was observed. The main cost driver for the model and sub-group analysis was length of stay. The sub-group analysis was based on a single comparative study. However, the MTAC received details of an unpublished audit of Thopaz+ which confirmed its efficacy in treating patients with pneumothorax. Thopaz+ received a positive recommendation in Medical Technologies Guidance 37.
\end{abstract}

\section{Key Points for Decision Makers}

Clinical evidence for Thopaz+ showed shorter drainage times and length of hospital stay, lower rates of chest drain re-insertion and higher rates of patient satisfaction when used in patients following pulmonary resection compared to conventional chest drainage. One comparative study of the use of Thopaz+ in patients with spontaneous pneumothorax was identified and showed shorter drainage times and length of hospital stay compared to conventional drainage.

The use of Thopaz+ in patients following pulmonary resection and patients with pneumothorax led to cost savings compared to conventional drainage. The main driver for cost savings was a reduction in length of hospital stay.

Megan Dale

megan.dale@wales.nhs.uk

Cardiff University, Cardiff, Wales, UK

2 Cedar, Cardiff and Vale University Health Board, Cardiff, Wales, UK

3 National Institute for Health and Care Excellence, Manchester, UK

\section{Introduction}

The Medical Technologies Evaluation Programme (MTEP) of the National Institute for Health and Care Excellence (NICE) evaluates innovative or new medical technologies. The programme provides support for National Health Service (NHS) technology adoption and generates guidance on diagnostic technologies and medical devices [1].

The process of completing these evaluations, known as Medical Technologies Guidance (MTG), has previously been described in detail [2]. Briefly, technologies are selected based upon company notifications on their medical device or technology; those selected have a scope published by NICE and this is followed by a submission by the company. The submission contains both clinical and economic evidence in order to make a case for adoption of the technology. External assessment centres review and critique the manufacturer's submission. The results of the review and critique are presented in an assessment report. The report is used, in addition to the manufacturer's submission, by the Medical Technologies Advisory Committee (MTAC) during its decision-making process. 
This paper reports on how Cedar's assessment report was used to inform MTG on the Thopaz+ portable digital system for managing chest drains. Cedar is a healthcare technology research centre formed through collaboration between Cardiff and Vale University Health Board and Cardiff University, and was responsible for producing the assessment report. Thopaz+ chest drain management systems are manufactured by Medela. This paper is part of a series that provides an insight into the development of NICE MTG [2].

\section{Background to the Condition and Technology}

Chest drains are required for the treatment of a number of procedures/conditions that affect the thoracic cavity. This includes, but is not restricted to, chest drainage following pulmonary resection and pneumothorax. In 2016-2017 there were 32,317 chest drain insertions of the pleural cavity, 10,821 drainages of the pleural cavity and 7336 instances of chest tubes of the pleural cavity requiring attention in England [3].

The insertion of chest drains has been recommended by NICE in their guideline for major trauma [4]. However, no previous guidance on chest drain management has been issued by NICE. The British Thoracic Society (BTS) has previously published guidelines on pleural disease [5]. The guidelines include recommendations on the use of chest drains including details on chest drain insertion, image guidance, aseptic technique, securing the drain, the drainage system used, and management of a chest drain. The guidelines do not specify the drainage system to be used for the chest drain; however, they do recommend that the drainage system should include a valve mechanism to prevent fluid or air from entering the pleural cavity.

A number of chest drain management systems exist. Many of these are simple in design and consist of a closed underwater seal bottle, which can be connected to suction if deemed appropriate. The underwater seal enables a visual check of air leak through the presence/absence of bubbles. The Thopaz+ chest drain management differs from more traditional drain management systems by using digital algorithms to monitor fluid drainage and air leakage in order to function. The device is able to maintain negative pressure and continuously monitor air leak. In addition, the device is portable and alerts users if safety issues, such as blocked tubing, arise. The manufacturer's submission contained a number of claimed benefits to the patients and included: reduced chest tube duration, reduced length of hospital stay, reduced rates of patient complications and higher patient satisfaction. The manufacturer claimed healthcare system benefits including: reduced hospital costs, increased convenience for doctors and nursing staff, improved chest drain management and better prediction of patient outcomes.

\section{Decision Problem (Scope)}

The manufacturer must define and follow a decision problem in order to focus their submission. The decision problem is presented in a PICO (population, intervention, comparator, outcomes) table. The PICO table generated by the manufacturer was consistent with the final scope which was published by NICE in May 2017 [6].

\subsection{Population}

For a population in their decision problem, the manufacturer identified "All people requiring a chest drain".

\subsection{Intervention}

The manufacturer identified Thopaz+ as the intervention. However, at the time of their submission there was no published evidence for the Thopaz+ device. All available evidence was for the company's earlier device, Thopaz. However, following discussions with the manufacturer, Cedar confirmed that the main method of action of the device has not changed so evidence for Thopaz is transferable and applicable to Thopaz+.

\subsection{Comparator}

The decision problem included the following comparators: underwater seal drains, chest drains involving a flutter valve and any other recognised mechanism or valve.

\subsection{Outcomes}

There were a number of outcomes listed by the manufacturer in their decision problem to provide evidence for their submitted claimed benefits. Clinical outcomes included duration of chest drain placement, incidence of chest drain re-insertion, fluid loss measurement, length of hospital stay, rate of complications and device-related adverse events and staff time. Patient satisfaction (including measures of patient discomfort) was the only patient-related outcome included by the company. The outcomes included by the manufacturer were used to assess their claimed benefits. From a healthcare perspective, a reduced length of hospital stay, reduced rate of complications and fewer adverse events would lead to reduced hospital costs. 


\section{Review of Clinical Effectiveness Evidence}

\subsection{Manufacturer's Review of Clinical Effectiveness Evidence}

Evidence submitted by the manufacturer aimed to match the scope [6]. A total of three studies were submitted by the manufacturer for consideration. One of the submitted studies was a randomised controlled trial (RCT) comparing the use of Thopaz to traditional "water-seal" suction drainage in patients following pulmonary resection [7]. The manufacturer also included one non-comparative study of the use of Thopaz in patients following pulmonary resection or lung biopsy [8], which was excluded by Cedar due to being out of scope. The remaining study was a non-comparative evaluation of the use of Thopaz in patients with pneumothorax [9]. The manufacturer included only studies that were freely available. Adverse events were searched for by the addition of extra search terms to the manufacturer's search strategy. No search for adverse events was carried out in the US Food and Drug Administration (FDA) Manufacturer and User Facility Device Experience (MAUDE) database. However, the manufacturer gave details of one adverse event reported through the Medicines and Healthcare products Regulatory Agency (MHRA).

\subsection{Cedar's Critique of Clinical Effectiveness Evidence}

The manufacturer carried out basic literature searches in Medline, Medline In Process, Embase and The Cochrane Library. However, it was unclear if all Cochrane Library databases were searched. The manufacturer assessed a total of 15 records at full text for eligibility to the submission and excluded a total of 9 studies as they were conference abstracts with insufficient data. The manufacturer also excluded 3 studies as they were not available free of charge online. Cedar did not feel this was a valid reason to exclude these studies, but the 3 studies were subsequently excluded by Cedar as they were conference abstracts with insufficient data. The company submitted critical appraisal checklists for all their included studies, and used the appropriate forms for each study type. The checklists were adapted from the Centre for Reviews and Dissemination [10].

Due to the simple search strategy used by the manufacturer, Cedar felt it was likely that relevant studies were not identified. Therefore, Cedar carried out its own literature search and reported its results using Preferred Reporting Items for Systematic Reviews and Meta-Analyses (PRISMA) methodology [11] (Fig. 1). In total, Cedar identified 11 studies relevant to the scope of this assessment [12-22] in addition to the two studies identified by the manufacturer, and included by Cedar [7, 9] to give a total of 13 studies.
Of the 13 included studies, there were six RCTs [7, 12, 14-16, 18], three observational comparative studies that used propensity matching for their analysis [20-22], three observational studies with no comparative element $[9,13$, 17] and one prospective comparative study [19]. The comparators in almost all of the RCTs and comparative observational studies were traditional analogue chest drainage systems that used wall-mounted suction. One of the RCTs used Thopaz at two different suction settings, and compared outcomes for these settings [12]. Eleven of the studies used the device in a patient population that had undergone pulmonary resection [7, 12-14, 16-22]. Two studies used the device in a patient population with pneumothorax $[9,15]$. A search of the FDA MAUDE database was not carried out by the manufacturer. Therefore, Cedar carried out its own search; in total, five adverse events were reported.

\subsection{Cedar's Conclusions on the Clinical Evidence}

A total of 13 studies were included by Cedar and of these, 2 were submitted by the company [7,9]. In most of the included comparative studies of Thopaz and conventional/ analogue drainage, patients who underwent pulmonary resection who were treated postoperatively with Thopaz had a shorter duration of chest tube drainage and a shorter hospital stay. Only one study reported a non-significant longer hospital stay for Thopaz [18]. No studies reported a longer duration of chest tube drainage with Thopaz. Two of the identified studies presented results for Thopaz used in patients with pneumothorax, one of which was comparative [15]. The results of this paper showed that both the duration of drainage and length of hospital stay were significantly shorter when using Thopaz. A single included study compared patient satisfaction between Thopaz and traditional drainage in patients undergoing pulmonary resection [7]. This study showed that patients treated with Thopaz had an improved ability to arise from bed, improved perceived system convenience, felt more comfortable being discharged home with the device if needed and fewer felt they would want to change the system compared with those treated with a traditional drainage device. The incidence of drain re-insertion was reported in 4 comparative papers where the incidence was non-significantly lower for Thopaz in 3 of these studies $[14,18,22]$ than traditional drainage. No drain re-insertions were required for patients treated with Thopaz or traditional drainage in one paper [16]. Cedar found no quantitative, comparative evidence measuring staff time saved when using Thopaz.

The evidence identified by Cedar was mainly from patients treated with Thopaz postoperatively following pulmonary resection. Cedar identified two studies where Thopaz was used for treatment of pneumothorax, one of which was a comparative study. This limited conclusions 


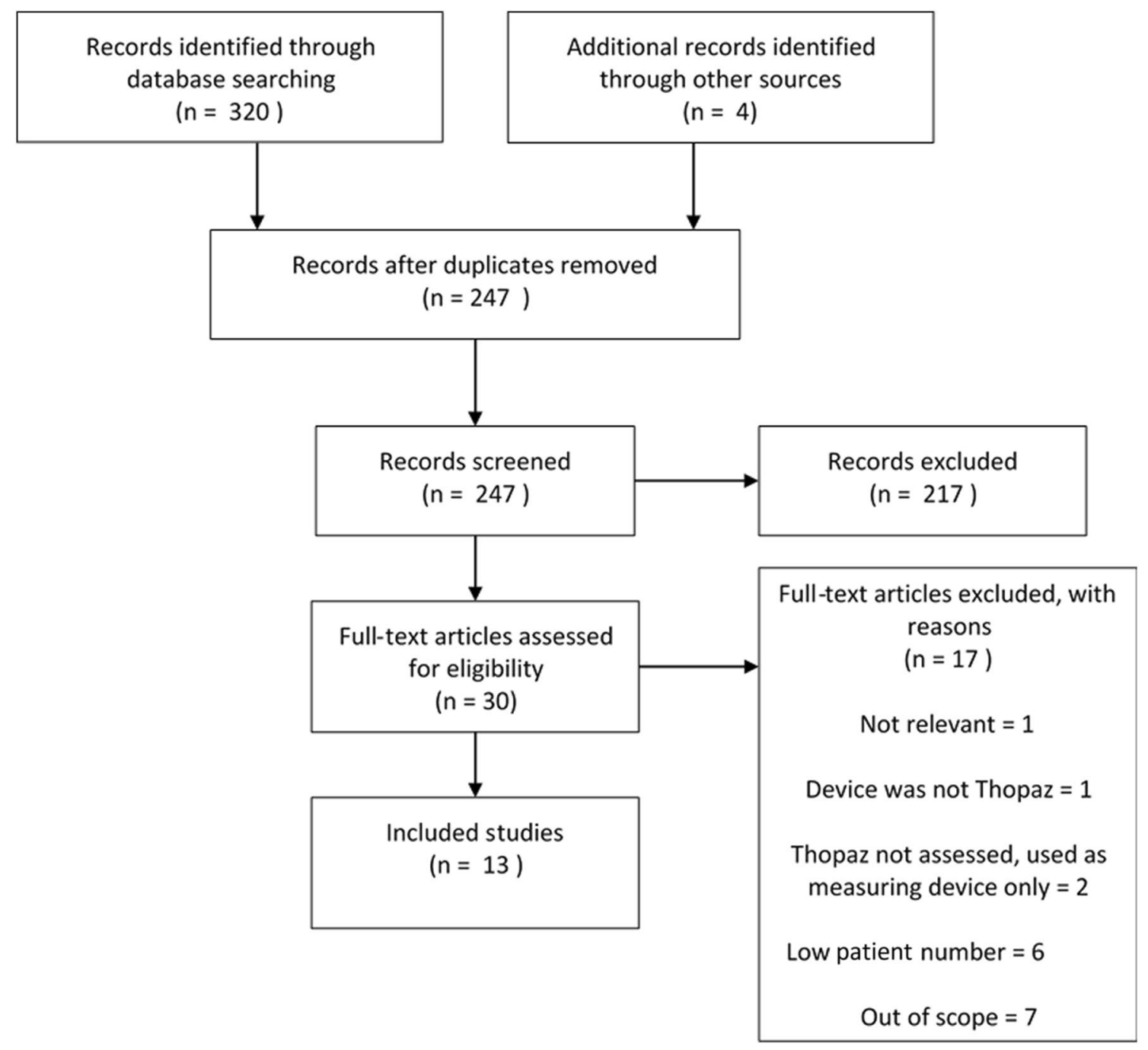

Fig. 1 PRISMA diagram showing Cedar's literature search results for Thopaz+. PRISMA Preferred Reporting Items for Systematic Reviews and Meta-Analyses

on the effectiveness of Thopaz in this patient group. The population identified in the scope was broad, but Cedar found no evidence for the use of Thopaz other than postoperative use in patients undergoing pulmonary resection or for the treatment of pneumothorax. Cedar did not find comparative evidence on the use of Thopaz for fluid loss measurement, an outcome in the scope. One identified paper looked at the use of Thopaz in a paediatric cohort [13]. Although non-comparative, this study was included to provide evidence for use in children in line with the subgroups defined in the scope. Further evidence is required to draw any conclusions on the effectiveness of Thopaz+ other than in patients undergoing pulmonary resection. It is also worth noting that centres included in each study followed different procedures for chest drain management. These are likely to affect the duration of chest tube drainage and in turn length of hospital stay. A summary of Cedar's included studies has been presented (Table 1).

\section{Economic Evidence}

\subsection{Manufacturer's Economic Submission}

The manufacturer's economic submission did not identify published economic evidence. The manufacturer submitted a de-novo economic model that followed a simple decision tree structure with a single decision node for Thopaz+ or standard drainage with wall suction. Clinical data for model inputs were obtained from a study of the use of Thopaz in patients following pulmonary resection that the company submitted as part of their clinical evidence [7]. 
Due to the choice of study, Cedar assumed the model was based on patients undergoing pulmonary resection. The manufacturer did not include data on patients with pneumothorax in their analysis. The technology considered was Thopaz+ and the comparator was standard drainage with wall suction in line with the scope. In their model the manufacturer made a key assumption that the cost of treatment with the comparator is zero, arguing that wall suction and all consumables are all readily available. The time horizon for the model was equal to the length of hospital stay.

Results of the manufacturer's economic analysis gave a base-case cost of $£ 1624.27$ for Thopaz+ and $£ 1659.83$ for standard drainage with suction. The manufacturer therefore estimated that using Thopaz+ would save $£ 35.56$ per patient when used in place of standard drainage with suction over the length of their hospital stay.

\subsection{Critique of Economic Evidence}

The manufacturer's submission did not contain any published economic evidence for Cedar to critique. However, the manufacturer utilised data on chest tube drainage from a study they submitted as part of their clinical evidence [7]. The manufacturer chose not to carry out cost modelling based on the study they submitted for patients with pneumothorax [9]. Cedar believed that the company's literature search for clinical evidence did not identify all available evidence on Thopaz. With a greater body of evidence, parameters such as chest tube duration could have been altered to reflect results in the literature.

\subsection{Critique of the De Novo Model}

The manufacturer's de novo model was a simple decision tree, but the manufacturer did not explicitly state which group of patients their economic model was based upon. Cedar assumed the model was based on patients undergoing pulmonary resection due to the study used to provide inputs into the model [7]. The manufacturer assumed there were no costs for the comparator. Cedar disagreed with this approach. There are costs associated with conventional drainage including the cost of the traditional device and its associated consumables. Including these costs would increase the cost saving of Thopaz+ in the submitted model. The model included training costs by using an estimate of the time taken for training coupled with staffing costs [23]. The company was highly conservative with their estimates of staffing costs, assuming that all physicians/surgeons were at consultant level and all nurses/ other healthcare staff were also at consultant level. Cedar felt that assuming all nurses/other healthcare staff were at consultant level was too conservative and increased the costs of Thopaz+. Not all of the available device pricing options were explored by the manufacturer. Deterministic sensitivity analysis carried out by the manufacturer did not take into account the difference in cost of rental for Thopaz+ and the different canister options available, in terms of canister size and whether or not the canister contains a solidifying agent. Finally, the manufacturer did not use expert advisers to inform their economic model or submission.

Following its critique, Cedar made a number of changes to the submitted model. Studies identified by Cedar were used to calculate means for duration of chest tube placement, length of hospital stay when using Thopaz+ and length of hospital stay when using traditional drainage. Cedar contacted expert advisers to determine a more robust figure for training costs. In addition, Cedar also included comparator costs, and calculated an incidence rate for chest drain reinsertion and its associated cost. Cedar conducted a number of scenario analyses including: the use of a purchased Thopaz+ machine for postoperative use in patients following pulmonary resection, the use of a rented Thopaz+ device and the use of a purchased Thopaz+ device for pneumothorax treatment.

Following its changes, Cedar's base-case showed a saving of $£ 111.34$ per patient when a rented Thopaz+ device was used postoperatively following pulmonary resection instead of conventional drainage. If a purchased Thopaz+ device was used in this patient population then savings were increased to $£ 124.76$ per patient if used instead of conventional drainage. If a rented or purchased Thopaz+ device was used for the treatment of pneumothorax instead of conventional drainage, then $£ 550.90$ and $£ 558.57$ per patient could be saved, respectively. In the base-case and all scenarios the main cost driver was length of hospital stay.

\section{NICE Guidance}

\subsection{Provisional Recommendations and Consultation}

The evidence submitted by the company and Cedar's critique of this evidence was presented to the MTAC who provided draft recommendations relating to Thopaz+ following their meeting in September 2017. These were as follows:

- The case for adopting Thopaz+ for managing chest drains is supported by the evidence. Thopaz+ can reduce drainage time and length of stay in hospital, and improves safety for people with chest drains. Its use may also improve clinical decision making through continuous, objective monitoring of air leaks and fluid loss. 


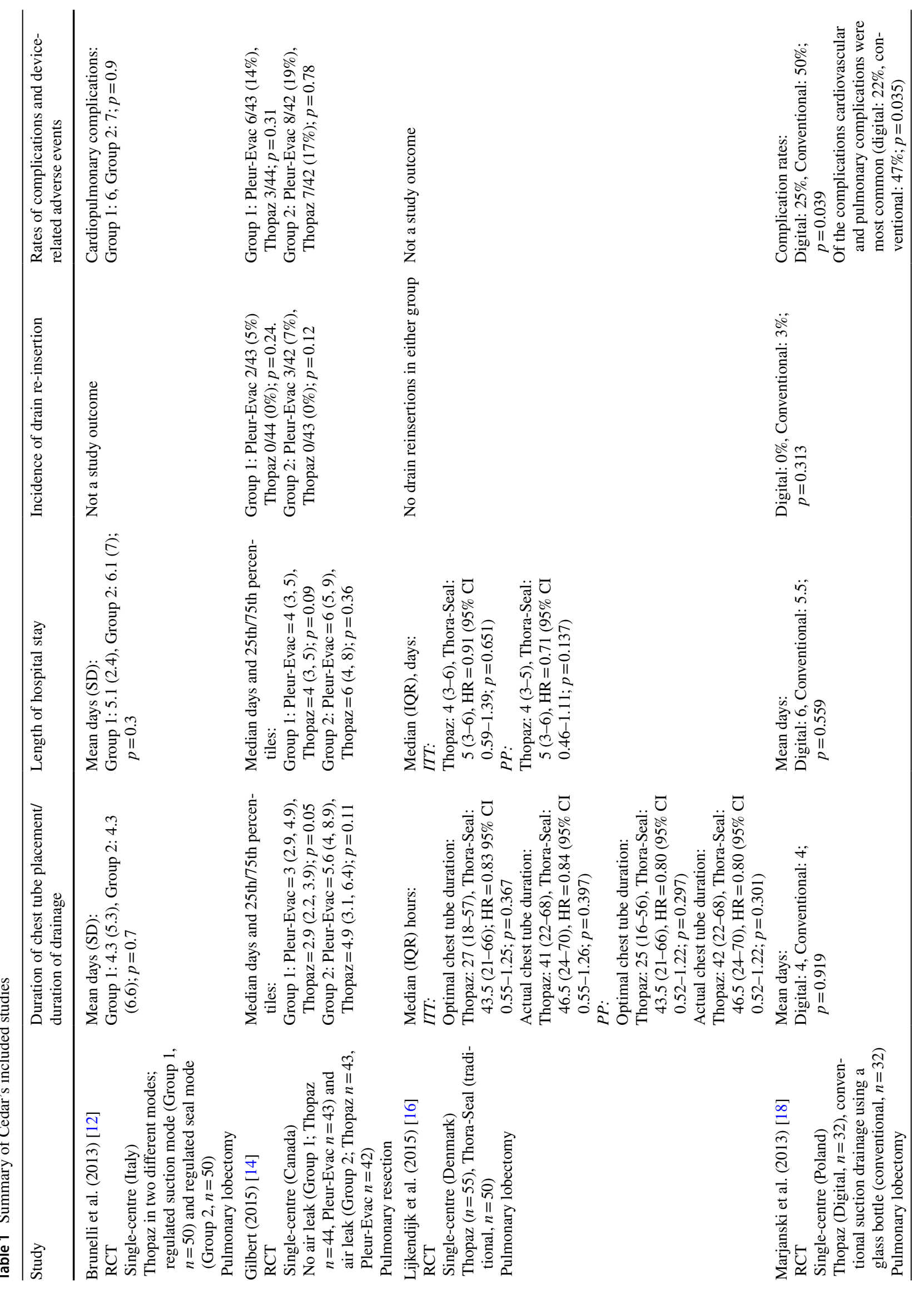




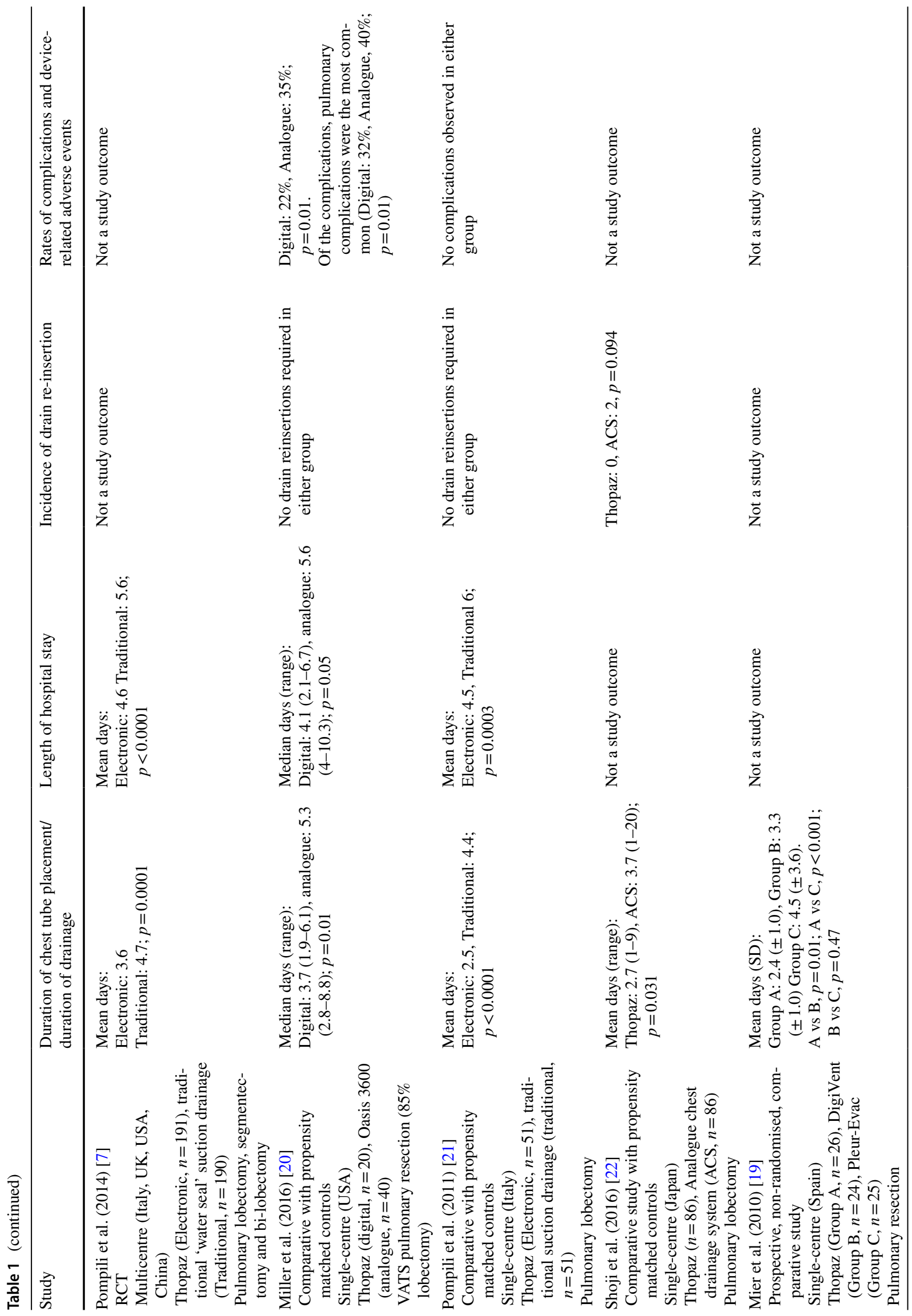




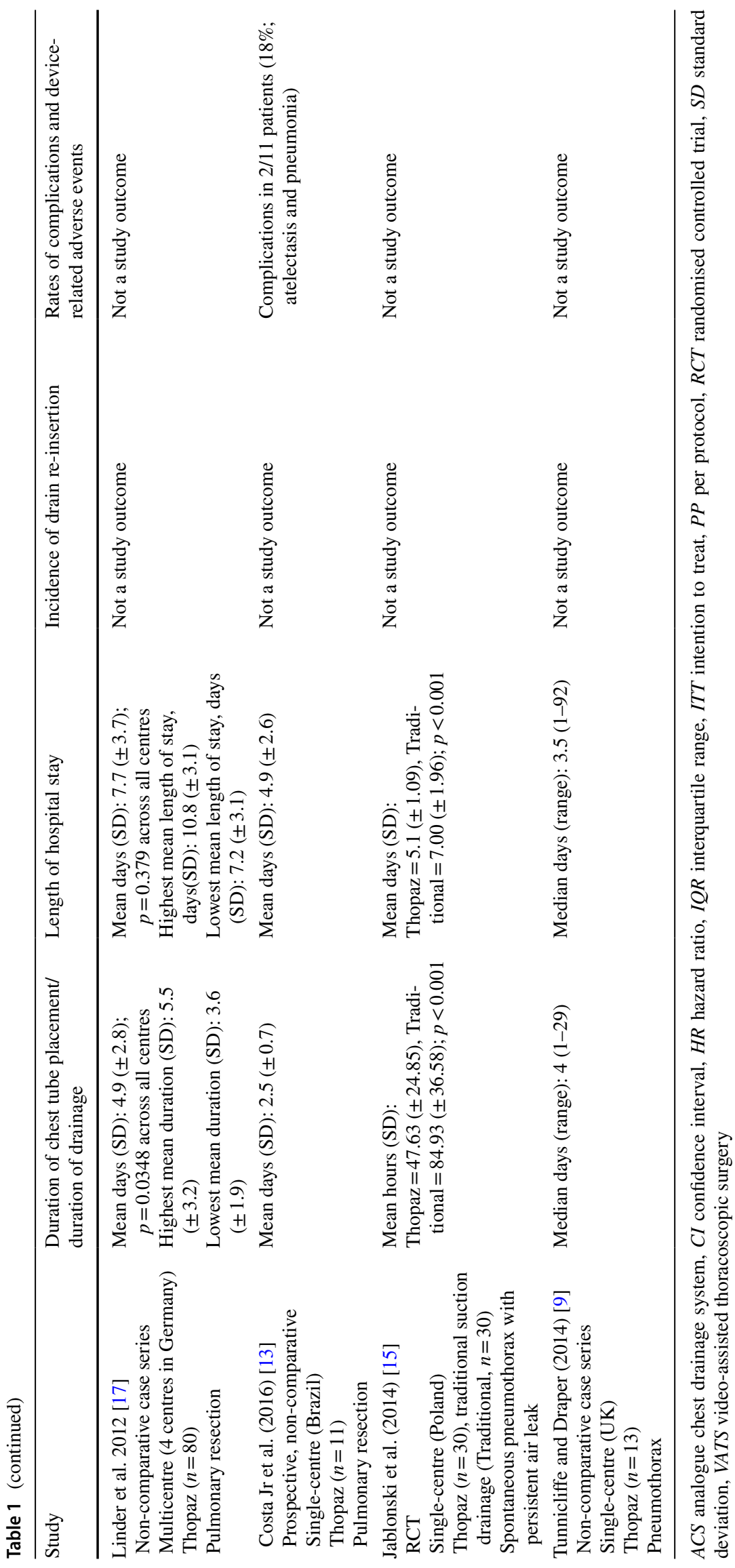


- Thopaz+ should be considered for people who need chest drainage after pulmonary resection or because of a pneumothorax. The system can increase patient mobility because it is portable. Staff find it more convenient and easier to use than standard wall suction.

- Cost modelling indicates that Thopaz+ is cost saving compared with standard wall suction in people who need chest drainage after pulmonary resection. The estimated saving is $£ 111.33$ per patient over their stay in hospital. These savings are mainly achieved through reduced length of stay in hospital.

\subsection{Final NICE Guidance}

During the consultation, NICE received 8 consultation comments from 4 consultees. The NICE guidance was updated to address a number of these comments. The recommendations had minor updates to better define the comparator as a conventional chest drain. Consultees suggested the evidence for Thopaz+ in pneumothorax was limited. The committee also received comments from an expert describing an unpublished audit of Thopaz+ use which confirmed the recommendations for use in pneumothorax. The Committee decided not to update the recommendations on pneumothorax.

\section{Key Challenges and Learning Points}

Through a combination of a lack of identified clinical evidence and no economic evidence coupled with weaknesses and limitations in the manufacturer's de novo economic model, Cedar was able to make a number of improvements. This included contacting clinical experts for advice on model inputs, the inclusion of comparator costs and modification of clinical parameters (e.g. length of hospital stay) based on published clinical evidence. In addition, Cedar carried out a number of scenario analyses to consider different procurement options and the use of Thopaz+ in the treatment of pneumothorax.

One of the key challenges faced by the MTAC was determining whether Cedar's scenario analysis of the use of Thopaz+ for the treatment of pneumothorax was reliable. The length of stay model input for this scenario came from a single comparative study [15]. In response, a clinical expert was able to share audit data from their NHS hospital which showed that Thopaz+ showed similar clinical advantages for patients following pulmonary resection and for the treatment of pneumothorax.

There was a lack of comparative evidence for the use of Thopaz+ in paediatric patients. Clinical experts noted that if the devices are available on wards they may be used safely for a range of patients. However, evidence for the use of Thopaz+ in a paediatric population is currently lacking.

\section{Conclusion}

Thopaz+ received a positive recommendation from NICE and should be considered for managing chest drains. Published evidence showed that Thopaz+ can reduce the duration of chest tube drainage and length of hospital stay. In its scenario analysis, Cedar showed that savings achieved using Thopaz+ instead of traditional drainage were greater for patients with pneumothorax than those achieved when using Thopaz+ postoperatively following pulmonary resection. Cedar highlighted uncertainty over the scenario analysis. However, audit data showed similar clinical advantages for the use of Thopaz+ in patients with pneumothorax and when used postoperatively following pulmonary resection.

Acknowledgements Cedar would like to acknowledge the following for providing expert clinical advice: Mr. Kostas Papagiannopoulos (Consultant Thoracic Surgeon, Leeds NHS Foundation Trust), Mrs. Catherine Plowright (Consultant Nurse in Critical Care, Medway NHS Foundation Trust) and Mrs. Jenny Mitchell (Senior Advanced Nurse Practitioner, Oxford University Hospital NHS Trust).

Author contributions JE and AR evaluated the clinical evidence, HM carried out literature searches, MD, JE and AR evaluated the economic evidence, MD created the economic model. JE, AR and GCR wrote the assessment report and PD wrote the assessment report overview both of which were presented to the Medical Technologies Advisory Committee. PD subsequently helped to develop the medical technologies guidance for NICE. GCR reviewed the full EAC report and this article, and can act as a guarantor for the overall content.

\section{Compliance with Ethical Standards}

Conflict of interest Cedar was funded by the NICE Medical Technologies Evaluation Programme for their work. At the time of completing the study JE, AR and HM were Cardiff University employees and have no conflict of interest. MD and GCR are NHS employees, and the NHS has a financial interest in the guidance on which this project is based. PD is a NICE employee and had no role in the production of the assessment report but contributed to the preparation of this manuscript. This summary of the Medical Technology Guidance was produced following the publication of the final guidance report. The article has not been externally peer reviewed by Applied Health Economics and Health Policy.

OpenAccess This article is distributed under the terms of the Creative Commons Attribution-NonCommercial 4.0 International License (http://creativecommons.org/licenses/by-nc/4.0/), which permits any noncommercial use, distribution, and reproduction in any medium, provided you give appropriate credit to the original author(s) and the source, provide a link to the Creative Commons license, and indicate if changes were made. 


\section{References}

1. National Institute for Health and Care Excellence. Medical Technologies Evaluation Programme: Methods Guide; 2017. https:// www.nice.org.uk/process/pmg33/chapter/introduction.

2. Campbell B, Campbell M. NICE medical technologies guidance: a novel and rigorous methodology to address a new health technology assessment challenge. Appl Health Econ Health Policy. 2012;10(5):295-7.

3. NHS Digital. Hospital Episode Statistics (HES) 2016-2017. from https://files.digital.nhs.uk/publication/7/g/hosp-epis-stat-admiproc-2016-17-tab.xlsx.

4. National Institute for Health and Care Excellence. Major trauma: assessment and initial management; 2016. https://www.nice.org. uk/guidance/ng39.

5. British Thoracic Society Pleural Disease Guideline Group. BTS pleural disease guideline; 2010. https://www.brit-thoracic.org. uk/document-library/clinical-information/pleural-disease/pleur al-disease-guidelines-2010/pleural-disease-guideline/.

6. National Institute for Health and Care Excellence. Thopaz+ portable digital system for managing chest drains: scoping document; 2017. https://www.nice.org.uk/guidance/mtg37/documents/final -scope. Accessed 3 July 2017.

7. Pompili C, Detterbeck F, Papagiannopoulos K, et al. Multicenter international randomized comparison of objective and subjective outcomes between electronic and traditional chest drainage systems. Ann Thorac Surg. 2014;98(2):490-6.

8. Rathinam S, Bradley A, Cantlin T, Rajesh P. Thopaz Portable Suction Systems in Thoracic Surgery: an end user assessment and feedback in a tertiary unit. J Cardiothorac Surg. 2011;6:59.

9. Tunnicliffe G, Draper A. A pilot study of a digital drainage system in pneumothorax. BMJ Open Respir Res. 2014;1(1):e000033.

10. Centre for Reviews and Dissemination. Systematic reviews. CRD's guidance for undertaking reviews in health care; 2008. https://www.york.ac.uk/media/crd/Systematic_Reviews.pdf.

11. Moher D, Liberati A, Tetzlaff J, Altman DG, The PRISMA Group. Reporting Items for Systematic Reviews and Meta-Analyses: the PRISMA statement. PLoS Med. 2009;6(7):e1000097.

12. Brunelli A, Salati M, Pompili C, et al. Regulated tailored suction vs regulated seal: a prospective randomized trial on air leak duration. Eur J Cardio-Thorac Surg. 2013;43(5):899-904.
13. Costa AD Jr, Bachichi T, Holanda C, Rizzo LA. An initial experience with a digital drainage system during the postoperative period of pediatric thoracic surgery. Jornal Brasileiro De Pneumologia: Publicacao Oficial Da Sociedade Brasileira De Pneumologia E Tisilogia. 2016;42(6):444-6.

14. Gilbert S, McGuire AL, Maghera S, et al. Randomized trial of digital versus analog pleural drainage in patients with or without a pulmonary air leak after lung resection. $\mathrm{J}$ Thorac Cardiovasc Surg. 2015;150(5):1243-9.

15. Jablonski S, Brocki M, Wawrzycki M, et al. Efficacy assessment of the drainage with permanent airflow measurement in the treatment of pneumothorax with air leak. Thorac Cardiovasc Surg. 2014;62(6):509-15.

16. Lijkendijk M, Licht PB, Neckelmann K. Electronic versus traditional chest tube drainage following lobectomy: a randomized trial. Eur J Cardio-Thorac Surg. 2015;48(6):893-8.

17. Linder A, Ertner C, Steger V, et al. Postoperative chest tube management: snapshot of German diversity. Interact Cardiovasc Thorac Surg. 2012;15(4):622-6.

18. Marjanski T, Sternau A, Rzyman W. The implementation of a digital chest drainage system significantly reduces complication rates after lobectomy - a randomized clinical trial. Kardiochirurgia I Torakochirurgia Polska. 2013;10(2):133-8.

19. Mier JM, Laureano M, Fibla JJ. The benefits of digital air leak assessment after pulmonary resection: prospective and comparative study. Cirugia Espanola. 2010;87(6):385-9.

20. Miller DL, Helms GA, Mayfield WR. Digital drainage system reduces hospitalization after video-assisted thoracoscopic surgery lung resection. Ann Thorac Surg. 2016;102(3):955-61.

21. Pompili C, Brunelli A, Salati M, et al. Impact of the learning curve in the use of a novel electronic chest drainage system after pulmonary lobectomy: a case-matched analysis on the duration of chest tubes. Interact Cardiovasc Thorac Surg. 2011;13(5):490-3.

22. Shoji F, Takamori S, Akamine T, et al. Clinical evaluation and outcomes of digital chest drainage after lung resection. Ann Thorac Cardiovasc Surg. 2016;22(6):354-8.

23. Curtis L, Burns A. Unit Costs of Health and Social Care 2016, Personal Social Services Research Unit (PSSRU), University of Kent, Canterbury; 2016. https://www.pssru.ac.uk/project-pages/ unit-costs/unit-costs-2016/. Accessed 2 Aug 2017. 